\title{
Reduction of Severity of Recurrent Psychotic Episode by Sustained Treatment with Aripiprazole in a Schizophrenic Patient with Dopamine Supersensitivity: A Case Report
}

\author{
Shigenori Tadokoro ${ }^{1,2}$, Naho Nonomura ${ }^{1,3}$, Nobuhisa Kanahara ${ }^{4}$, Kenji Hashimoto ${ }^{5}$, Masaomi lyo ${ }^{1}$ \\ ${ }^{1}$ Department of Psychiatry, Chiba University Graduate School of Medicine, Chiba, ${ }^{2}$ Health Administration Center, Muroran Institute of \\ Technology, Hokkaido, ${ }^{3}$ Department of Biomedical Ethics, Graduate School of Medicine, University of Tokyo, Tokyo, ${ }^{4}$ Division of Medical \\ Treatment and Rehabilitation, ${ }^{5}$ Division of Clinical Neuroscience, Chiba University Center for Forensic Mental Health, Chiba, Japan
}

\begin{abstract}
Dopamine supersensitivity psychosis (DSP) is a type of acute exacerbation of recurrent psychosis caused by long-term treatment with antipsychotics in schizophrenic patients. Although DSP is exceedingly troublesome for clinicians, effective treatment has not yet been established. Based on clinical research and our animal study, we hypothesize that aripiprazole, an atypical antipsychotic, may reduce the exacerbation of recurrent psychotic episodes. We report the case of a 46-year-old female who suffered from schizophrenia with DSP. In this case, sustained treatment with a high dose of aripiprazole gradually reduced the severity of her recurrent psychotic episodes. In conclusion, sustained treatment with aripiprazole may reduce the exacerbation of recurrent psychotic episodes in schizophrenic patients with DSP, and may be an effective treatment of DSP.
\end{abstract}

KEY WORDS: Antipsychotic agents; Disease exacerbation; Receptors, dopamine D2; Recurrence; Schizophrenia.

\section{INTRODUCTION}

Dopamine supersensitivity psychosis (DSP) is a type of acute exacerbation of recurrent psychosis in schizophrenic patients with excessive sensitivity to dopamine, caused by long-term treatment with antipsychotics. ${ }^{1)}$ Furthermore, it has been estimated that more than half the cases of treatment-resistant schizophrenia might be related to DSP. ${ }^{2)}$ Because DSP is associated with tolerance development for antipsychotics and/or vulnerability to minor stress, ${ }^{1)}$ it is exceedingly troublesome for clinicians. Nevertheless, no effective treatment has yet been established for DSP.

On the other hand, some clinical studies on the treatment of patients with schizophrenia have suggested that aripiprazole, one of the atypical antipsychotics, might lower the risk of relapse and prevent the exacerbation of psychotic symptoms more efficiently than other antipsychotics. ${ }^{3,4)}$ Furthermore, in a previous study where we used an animal model of DSP, results suggested that aripiprazole may

\footnotetext{
Received: February 24, 2016 / Revised: May 21, 2016

Accepted: May 23, 2016

Address for correspondence: Shigenori Tadokoro, MD, PhD Health Administration Center, Muroran Institute of Technology,

27-1. Mizumoto-cho, Muroran, Hokkaido 050-8585, Japan

Tel: +81-143-46-5851, Fax: +81-143-46-5850

E-mail: tadokoroshigenori@gmail.com
}

have not only preventive but also therapeutic effects on DSP through the reduction of excessive sensitivity to dopamine. ${ }^{5)}$ Based on these findings, we hypothesize that aripiprazole may reduce the exacerbation of recurrent psychotic episodes in schizophrenic patients with DSP. Here we report a case study supporting our hypothesis. Incidentally, informed consent was obtained from the patient for publication of this case report.

\section{CASE}

The patient is a 46-year-old married Japanese woman with a long history of schizophrenia. Her psychotic symptoms, which include delusions of persecution and thought broadcasting, first appeared at age 35 years. At the time, she underwent ambulatory treatment with 4-mg risperidone, and as a result did not experience another psychotic episode for about 10 years. Nevertheless, one year ago, her first recurrence of a psychotic episode was triggered by a trifling family problem, and her psychotic symptoms increased in severity to the point that she required hospitalization. On this occasion, an additional dose of risperidone and doses of other antipsychotics barely cured her symptoms, and she was discharged from the hospital after

(c) This is an Open-Access article distributed under the terms of the Creative Commons Attribution Non-Commercial License (http://creativecommons.org/licenses/by-nc/4.0) which permits unrestricted non-commercial use, distribution, and reproduction in any medium, provided the original work is properly cited. 


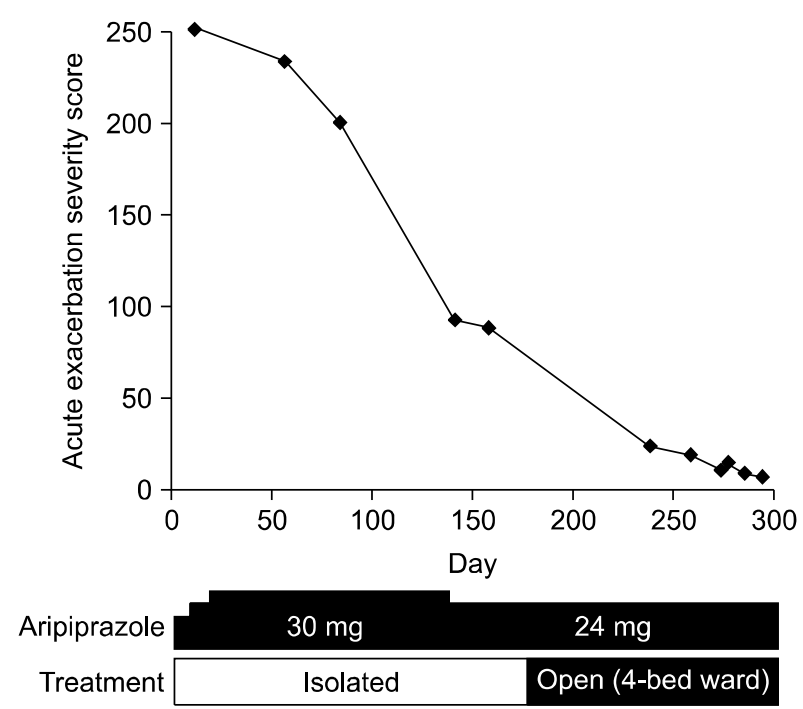

Fig. 1. This figure shows the relationship between Acute Exacerbation Severity (AES) scores of 11 recurrent psychotic episodes and the duration of sustained treatment with aripiprazole. AES scores were defined as the Excited Component of the Positive and Negative Syndrome Scale scores multiplied by the episode duration in 0.5-day units. As may be seen, the AES score of each episode decreased along with the time course of sustained treatment with aripiprazole.

two months. Three months later, however, her psychotic symptoms relapsed without any apparent trigger, and this time, neither the maximum dose of risperidone nor other antipsychotics were able to control her psychotic episodes. Therefore, she was admitted to Chiba University Hospital for specialized treatment.

After admission, she was mainly treated with a high dose (24 mg or more) of aripiprazole, together with a variable dose of olanzapine (5-10 mg) or quetiapine (200-700 $\mathrm{mg}$ ) for a short period of time. Over approximately two months, similar episodes of acute exacerbation of psychotic symptoms were repeated and continued for about a week each time. She was violently excited, refused medication, and demanded to be discharged. As a result, she was forced to live in an isolated room. Three months after the start of sustained treatment with the high dose of aripiprazole, however, the duration of exacerbation episodes became shorter, while the severity of her psychotic symptoms were gradually alleviated. Six months after the start of the treatment, episodes of exacerbation ended within half a day, and her psychotic symptoms became insignificant. She was able to move to an open room (4-bed ward). Finally, ten months after the start of treatment, she was discharged from our hospital without any significant psychotic episodes.
In order to quantify the severity of acute exacerbation episodes, we used the Excited Component of the Positive and Negative Syndrome Scale (PANSS-EC). ${ }^{6}$ We defined the Acute Exacerbation Severity (AES) score as the PANSS-EC score multiplied by the duration of the episode in 0.5-day units, and examined the AES score of each episode. We recorded the AES scores of the patient during the 305 days of hospital treatment (Fig. 1), and detected 11 episodes of acute exacerbation. As can be seen from the figure, the AES score of each episode decreased along with the time course of sustained treatment with aripiprazole.

\section{DISCUSSION}

We think that this patient suffered from DSP caused by long-term treatment with risperidone. Therefore, she had repeated similar episodes of acute exacerbation without any apparent trigger, owing to her excessive dopamine sensitivity. We consider that sustained treatment with a high dose of aripiprazole gradually reduced the severity of her recurrent psychotic episodes through the reduction of this excessive sensitivity to dopamine. It is known that chronic treatment with $\mathrm{D}_{2}$ dopamine receptor antagonists, such as haloperidol, up-regulates the density of $\mathrm{D}_{2}$ receptors, and leads to DSP. ${ }^{7)}$ Based on the results of our animal study and our theoretical findings, we think that aripiprazole can down-regulate the density of $\mathrm{D}_{2}$ receptors and ameliorate DSP, as a result of its partial-agonistic effects on the $\mathrm{D}_{2}$ receptors. ${ }^{8)}$ Therefore, we believe that sustained treatment with a high dose of aripiprazole might have down-regulated the density of the patient's $\mathrm{D}_{2}$ receptors, and as a result, reduced the exacerbation of her recurrent psychotic episodes.

Because aripiprazole yields excessive dopaminergic neurotransmission in patients with DSP due to increased $\mathrm{D}_{2}$ receptor density, a transient worsening of psychosis can appear following the switch to aripirazole. ${ }^{5,9)}$ In fact, the results of a clinical survey suggested that patients with DSP were more likely to experience psychotic worsening following the switch to aripiprazole, especially when the patients had undergone relatively slow titration, i.e., a low initial dose with a gradual increase of aripiprazole. ${ }^{10)}$ However, our patient had undergone a quick titration of aripiprazole. We therefore suspect that the excessive dopaminergic neurotransmission can gradually down-regulate the density of a patient's $\mathrm{D}_{2}$ receptors due to compensatory systems induced over the time course of treatment, without causing any transient worsening of psychosis.

In this case, sustained treatment with aripiprazole may 
have reduced the exacerbation of recurrent psychotic episodes in a schizophrenic patient with DSP. It might be an effective treatment of DSP. However, well-designed, randomized, double blind, placebo-controlled studies using a large sample would be needed to confirm this.

\section{Acknowledgments}

Funding for this study was provided by Department of Psychiatry, Graduate School of Medicine, Chiba University.

\section{REFERENCES}

1. Chouinard G. Severe cases of neuroleptic-induced supersensitivity psychosis. Diagnostic criteria for the disorder and its treatment. Schizophr Res 1991;5:21-33.

2. Chouinard G, Chouinard VA. Atypical antipsychotics: CATIE study, drug-induced movement disorder and resulting iatrogenic psychiatric-like symptoms, supersensitivity rebound psychosis and withdrawal discontinuation syndromes. Psychother Psychosom 2008;77:69-77.

3. Gorwood P1. Meeting everyday challenges: antipsychotic therapy in the real world. Eur Neuropsychopharmacol 2006;16 Suppl 3:S156-S162.

4. Croxtall JD. Aripiprazole: a review of its use in the management of schizophrenia in adults. CNS Drugs 2012;26. 155-183.
5. Tadokoro S, Okamura N, Sekine Y, Kanahara N, Hashimoto $\mathrm{K}$, Iyo M. Chronic treatment with aripiprazole prevents development of dopamine supersensitivity and potentially supersensitivity psychosis. Schizophr Bull 2012;38:1012-1020.

6. Montoya A, Valladares A, Lizán L, San L, Escobar R, Paz S. Validation of the Excited Component of the Positive and Negative Syndrome Scale (PANSS-EC) in a naturalistic sample of 278 patients with acute psychosis and agitation in a psychiatric emergency room. Health Qual Life Outcomes 2011;9:18.

7. Samaha AN, Seeman P, Stewart J, Rajabi H, Kapur S. "Breakthrough" dopamine supersensitivity during ongoing antipsychotic treatment leads to treatment failure over time. J Neurosci 2007;27:2979-2986.

8. Iyo M, Tadokoro S, Kanahara N, Hashimoto T, Niitsu T, Watanabe $\mathrm{H}$, et al. Optimal extent of dopamine D2 receptor occupancy by antipsychotics for treatment of dopamine supersensitivity psychosis and late-onset psychosis. J Clin Psychopharmacol 2013;33:398-404.

9. Di Lorenzo R, Amoretti A, Forghieri M, Fiorini F, Genedani S, Rigatelli M. Aripiprazole: effectiveness and safety under naturalistic conditions. Exp Clin Psychopharmacol 2007;15: 569-575.

10. Takase M, Kanahara N, Oda Y, Kimura H, Watanabe H, Iyo M. Dopamine supersensitivity psychosis and dopamine partial agonist: a retrospective survey of failure of switching to aripiprazole in schizophrenia. J Psychopharmacol 2015;29: 383-389. 